\title{
The High Latitude Ionospheric Convection Pattern
}

\author{
R. A. HEELIS \\ Center for Space Sciences, Physics Programs, University of Texas at Dallas, Richardson, Texas, U.S.A.
}

(Received September 1, 1990; Revised March 18, 1991)

\begin{abstract}
Simple examination of models of the magnetic and electric field indicate that both direct connection with the interplanetary field and connection to the lowlatitude boundary layer flow are required to explain many of the repeatable features in the observations of high latitude ionospheric motions. During times of southward IMF, these two sources of the electric field may not be easily discernible and during time of northward IMF the details of the magnetic field geometry are important in determining the geometry of the ionospheric convection cells. When both these sources of the electric field are considered, a transition from southward to northward IMF leads to distorted two-cell convection patterns that can contain multiple cell configurations.
\end{abstract}

\section{Introduction}

The past several years have seen considerable progress in the observational description of the high latitude ionospheric convection pattern (e.g. HEELIS, 1984; HolT et al., 1987; HEPPNER and MAYNARD, 1987) and in the development of theoretical ideas addressing the nature of the drivers for the convection electric field (CROOKER, 1979; LYONS, 1985; REIFF and BURCH, 1986; MOSES et al., 1988; LOCKWOOD et al., 1990). All these studies agree on the existence of a two cell convection pattern during periods of southward interplanetary magnetic field (IMF) with varying degrees of importance attached to different drivers. Perhaps the most variety of interpretations, both observationally and theoretically, exist in the description of the convection pattern and its drivers for conditions of northward IMF.

Various terms have been used to describe the electric field sources for the high latitude convection pattern. Here we consider three sources that are related in specific ways. The process of interconnection between the IMF and the geomagnetic field provides a means of applying the solar wind emf to the ionospheric plasma along highly conducting field lines. We will not discuss the theoretical concepts involved in the nature or efficiency of the interconnection process. The electric field originating from a magnetohydrodynamic generator in the low latitude boundary layer may also be applied to the high latitude ionospheric plasma along highly conducting magnetic field lines. This electric field is derived from the plasma in the low-latitude boundary layer, which is flowing antisunward in a manner similar to that seen in the magnetosheath. Again, we will not discuss in detail the processes by which such motion is induced in the low-latitude boundary layer, but simply use the term viscous interaction to describe it.

These two electric field sources also produce a potential difference across the closed field line region of the magnetosphere encompassing the plasma sheet. That resulting from the low latitude boundary layer will always manifest itself as a dawn to dusk electric field, 
whereas that from interconnection with the IMF will depend upon the magnetic field geometry. In general a dawn to dusk field will result for southward directed IMF and a dusk to dawn electric field will result for northward IMF. The plasma sheet particles will themselves determine the distribution of the electric field and it may to some degree be considered as separate from the externally applied potential difference (cf. LOCKWOOD et al., 1990). We will refer to this electric field as the plasma sheet field. These electric fields are communicated to the ionosphere by field-aligned currents, which are then closed in a manner that is determined in part by the ionospheric conductivity. The electric fields from these different sources are therefore coupled through the ionosphere. Thus, for southward IMF it might be convenient to think of direct connection of the solar wind electric field as producing only antisunward motion of the ionospheric plasma, but in fact ionospheric current continuity will require that sunward plasma convection take place at lower latitudes. We have assigned this electric field source to plasma flow in the plasma sheet, but its ultimate source is the solar wind electric field and its distribution is intimately related to the ionospheric conductivity distribution (HAREL et al., 1981). The objective is to describe a self consistent distribution of electric and magnetic fields that will provide a framework for interpretation of ionospheric plasma drift and electric field measurements.

\section{Southward Interplanetary Magnetic Field}

When the IMF has a southward component both observational and theoretical work indicate the existence of a two-cell convection pattern with antisunward convection at the highest latitudes separated from sunward convection in the auroral zones by a well defined convection reversal boundary. Poleward of the convection reversal boundary, the geometry of the convection cells is determined largely by the IMF $y$-component (HEPPNER, 1977; HEPPNER and MAYNARD, 1987). In the northern hemisphere, when $B_{y}$ is positive, the sunward flow in the duskside auroral zone continues across local noon and predominantly across the dawnside at highest latitudes. When $B_{y}$ is negative the dawnside auroral zone flow continues across local noon and predominantly across the duskside at highest latitudes. These flow configurations do not show symmetry about the noon-midnight meridian (e.g. HolT et al., 1987).

For a southward directed IMF the magnetosheath and solar wind flow produce a dawn to dusk electric field. Direct connection to this field along open field lines to the ionosphere will produce antisunward flow at high latitudes. For this same configuration of the IMF the plasma sheet electric field from all sources will result in a net plasma flow toward the earth that corresponds to antisunward flow at high latitudes and sunward flow at lower latitudes in the ionosphere. Two transitions in the magnetic field topology take place along the resulting closed loop ionospheric plasma flow trajectories. One occurs across what we call a merging region; where the plasma flows from dayside closed geomagnetic field lines that map to the dayside magnetopause to open field lines that directly connect to the IMF and its associated electric field. The other occurs in the reconnection region where plasma flows from open field lines to closed magnetic field lines that thread the plasma sheet.

In general all models agree that the merging region has an ionospheric signature located on the dayside of the boundary enclosing a region of open field lines and the signature of the reconnection region in the magnetotail appears around the nightside of this boundary. The resulting ionospheric convection pattern and magnetic field topology is shown in Fig. 1. In this figure the dashed line represents the boundary enclosing a region of open field lines. The 

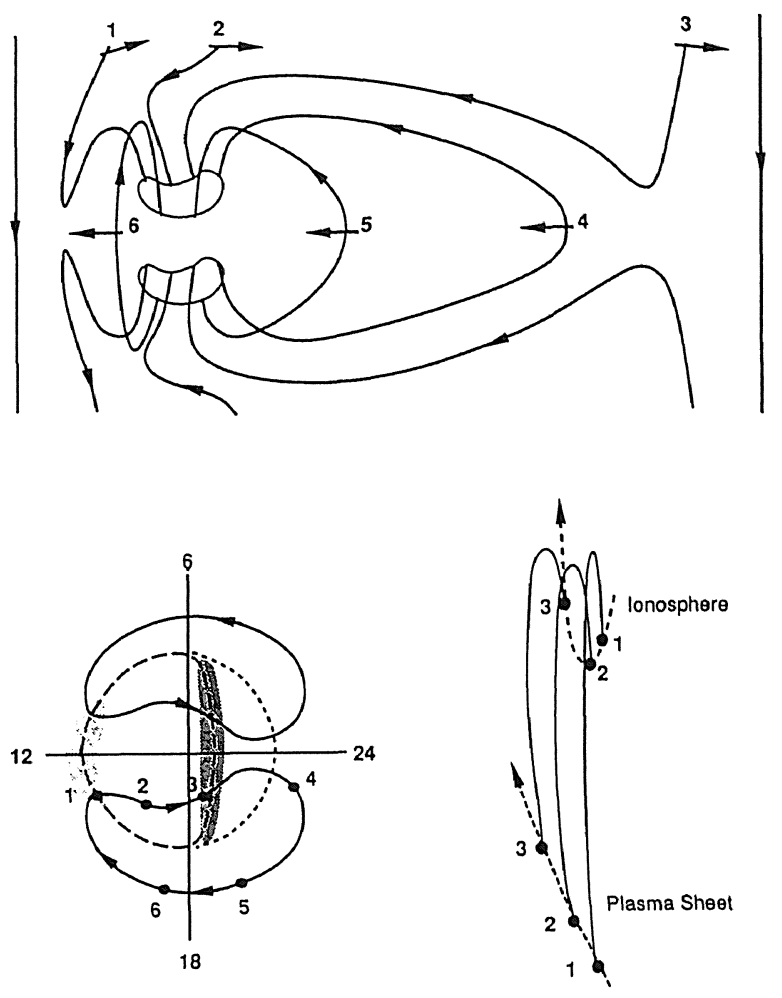

Fig. 1. Schematic representation of the magnetic field geometry and ionospheric flow resulting from direct connection with a southward IMF. The numbered arrows show the direction of the magnetospheric flow associated with the corresponding locations on the ionospheric convection pattern. The association between sunward flow in the plasma sheet and sunward and antisunward flow in the ionosphere is also illustrated.

field lines at latitudes below this boundary are closed. The lightly shaded region is the ionospheric signature of the merging region and the heavily shaded region is the ionospheric signature of a nightside reconnection region.

The models of CROOKER (1979), LYONS (1985), MOSES et al. (1988) and REIFF and BURCH (1986), differ in the degree to which they address the details of how and where the flow across the merging region takes place and the role of ionosperic conductivity gradients in determining the ionospheric flow. In the field addition model of LYONS, the potential derived from the solar wind along the line separating open and closed field lines, maps to a point in the ionosphere. No electric field exists at this line (since it would otherwise have to be infinite in the ionosphere) and the nature of the transition of the flow from open the closed field lines, or the convective flow on closed field lines is not addressed. The model of MOSES et al. (1988), quite accurately reproduces the geometry of the observed convection cells for southward IMF by specifying the potential distribution along a spiral shaped boundary enclosing the region of open field lines. However, the nature of the mapping of this boundary to the magnetopause or the value of the potential along it are not addressed. The models of 
REIFF and BURCH (1986) and CROOKER (1988) are more specific about the nature of the dayside merging region at the magnetopause and in fact map an image of this region, a merging line, to the ionosphere. They succesfully predict the $B_{y}$ dependence of the ionospheric flow pattern at high latitudes during times of southward IMF (e.g. HEELIS, 1984; HoLT et al., 1987). While these models address the location of the merging region in varying degrees of detail, none of them discuss the location of the reconnection region with any rigor.

In Fig. 1 a general description of the merging region as an arc along the boundary enclosing open field lines has been adopted to emphasize features that are not dependent on the level of detail presented in many of the models. Some part of the boundary enclosing the open field line region is shown as an electric equipotential so that no plasma flows across it where there is no shading. Note that the region of antisunward convection need not define the region of open field lines. This point is emphasized by adding a dotted line signifying a convection reversal boundary. The electric field in the plasma sheet, in this case derived from the application of the solar wind electric field across the region of closed field lines in the ionosphere, produces sunward plasma motion at all points in the plasma sheet. In the center of the plasma sheet where magnetic field lines extend into a tail like configuration the corresponding flow in the ionosphere is antisunward. Sunward flow in the ionosphere and the plasma sheet is appropriately mapped between regions that are closer to the earth. This association between sunward flow in the plasma sheet and both sunward and antisunward flow in the ionosphere is illustrated in the lower right panel of Fig. 1.

The relationship between plasma flows in the ionosphere and magnetosphere is complicated by an essential time dependence in the phenomena being described and by the existence of field aligned potential differences that may exist on field lines connecting the outer magnetosphere and the ionosphere. SISCOE and HUANG (1985) have shown for example, that if the potential distribution, around the boundary enclosing open field lines is not a constant then this boundary may move at the same velocity as the plasma and be an adiaroic line. LOCKWOOD et al. (1990) emphasize that the electric field sources from direct connection with the solar wind and from the plasma sheet can be quite independent if temporal changes in the magnetic field are accounted for. Thus the ionospheric plasma flow and the shape of the boundary enclosing the open field line region will respond to a superposition of these electric field sources. In the schematic representation of Fig. 1, there are no closed field lines tailward of the reconnection line. This is a consequence of the electric field mapping process invoked from the earthward flowing plasma in the plasma sheet. This concept is complicated by the recent finding of HEIKKILA (1988) that the flow across the distant tail plasma sheet is on average directed away from the earth at all locations. The electric field associated with this flow in the center of the plasma sheet would drive the ionospheric plasma sunward. This electric field source must therefore be isolated from the ionosphere by an effective potential difference along the magnetic field. At locations where the existence of field-aligned electric fields and temporal changes in the magnetic field prevent the direct application of the electric field in the outer magnetosphere to the ionosphere, the ionospheric motion will be determined by the current closure properties of the ionosphere rather than the potential distribution in the outer magnetosphere to which the magnetic field lines connect. The problem of where the current closes in the magnetosphere has not yet been addressed.

Like interconnection to the solar wind electric field in the case of soutward IMF, viscous interaction will produce antisunward plasma motion at the highest latitudes in the ionosphere. This plasma flow is on closed field lines and is always antisunward regardless of the orientation of the IMF. Again, the plasma sheet electric field will produce both 

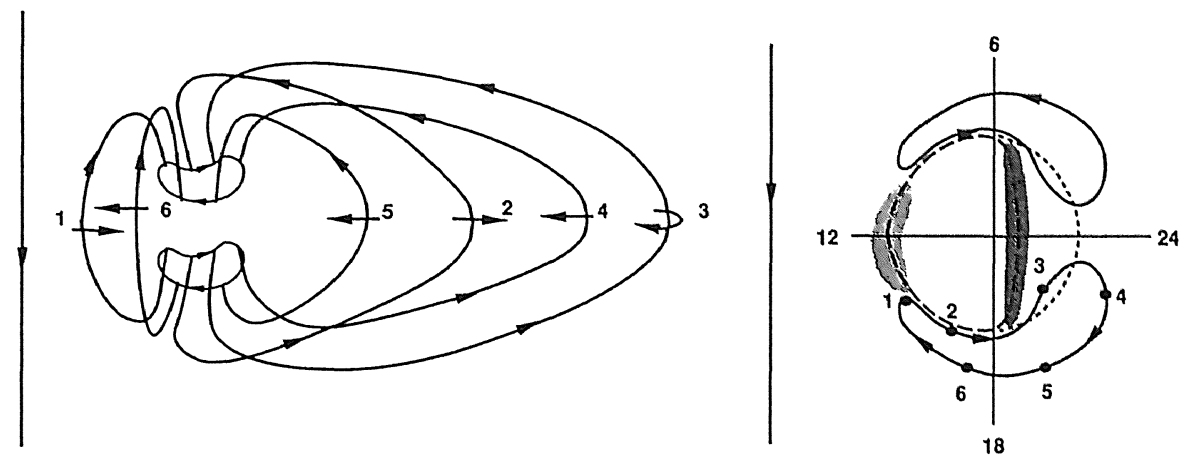

Fig. 2. Schematic representation of the magnetic field geometry and ionospheric flow associated with connection to the low-latitude boundary layer flow. Numbered arrows and heavy dots associate corresponding regions in the magnetosphere and ionosphere.

antisunward and sunward convection in the ionosphere and a closed loop convection pattern will result in which no transitions in magnetic field topology exist. The magnetic field configuration and ionospheric convection pattern in such a case is shown in Fig. 2. Here antisunward flow of closed field lines takes place in the ionosphere and in the low latitude boundary layers just inside the magnetopause. The sunward flow on closed field line is identical to that shown in Fig. 1.

During periods of southward IMF the relative roles of direct connection to the solar wind electric field and viscous interaction are difficult to assess. Both these drivers produce a twocell convection pattern with the same sense of circulation. In order to distinguish between these two drivers the boundary between open and closed field lines in the ionosphere must be located. Note, however, that a contribution from viscous interaction does not change the essential features of the plasma convection pattern during southward IMF. A region of open field lines contained within the dashed boundary has been retained in Fig. 2 to emphasize two points. First, without knowledge of the magnetic field topology at high latitudes the convection patterns shown in Figs. 1 and 2 could be quite indistinguishable. Second, both viscous interaction and dayside merging can simultaneously contribute to the high latitude convection pattern.

During periods of southward IMF the influence of the $y$-component of the IMF has been adequately described. The strong influence of the $y$-component of the IMF on the configuration of the dayside ionospheric flow (HEELIS, 1984; HOLT et al., 1987; HEPPNER and MAYNARD, 1987) suggests that direct connection is a significant driver for the ionospheric convection pattern. Furthermore, consideration of the magnetic field geometry associated with the interconnected geomagnetic and interplanetary fields successfully explains the observed asymmetry of the convection trajectories in the dayside high latitude ionosphere (e.g. CROOKER, 1979; LYONS, 1985) Nevertheless, this author knows of no unequivocally acceptable method of determining whether magnetic field lines in the ionosphere are open or closed. Thus it seems prudent not to eliminate any of the convection drivers from consideration, or to assess their relative importance without further study. 


\section{Northward Interplanetary Magnetic Field}

When the IMF has a northward component the identification of large scale convection patterns at high latitudes is complicated by the permanent presence of small scale structure with amplitudes as large as the large scale features. Despite this complication, satellite observations of the ionospheric electric field (BURKE et al., 1979; HEPPNER and MAYNARD, 1987), the horizontal current (ZANETTI et al., 1984), and the ion drift velocity (HEELIS et al., 1986), are generally consistent with three or more regions of sunward flow and two or more regions of antisunward flow that are alternately adjacent. The largest spatial scale signature of this type have been cited as evidence for the existence of four-cell (BURKE et al., 1979) and distorted two-cell convection patterns (HEPPNER and MAYNARD, 1987).

For northward IMF there exist a variety of models predicting the location of the merging region in the ionosphere and the topology of the magnetic field lines on which the plasma convects. Although further study is required it appears likely that both sunward and antisunward convection on open field lines must be understood. In the models of RUSSELL (1972), CROOKER (1979), LYONS (1985) and REIFF and BURCH (1986) this feature occurs on open field lines originating in the northern hemisphere polar cap and extending into the interplanetary medium to the south of the planet. Figure 3 schematically shows the ionospheric convection trajectory and the magnetic field topology associated with this configuration. The illustrated process has been called flux tube recirculation by CROOKER (1979) and lobe cell convection by REIFF and BURCH (1986). In the schematic it is assumed that a closed convection trajectory entirely on open field lines can exist in the ionosphere. Then no merging or reconnection signatures exist as we have defined them and a conceptual picture arises in which closed loop circulation in the ionosphere is not associated with a similar closed loop circulation in the outer magnetosphere. Here, for example, the simple

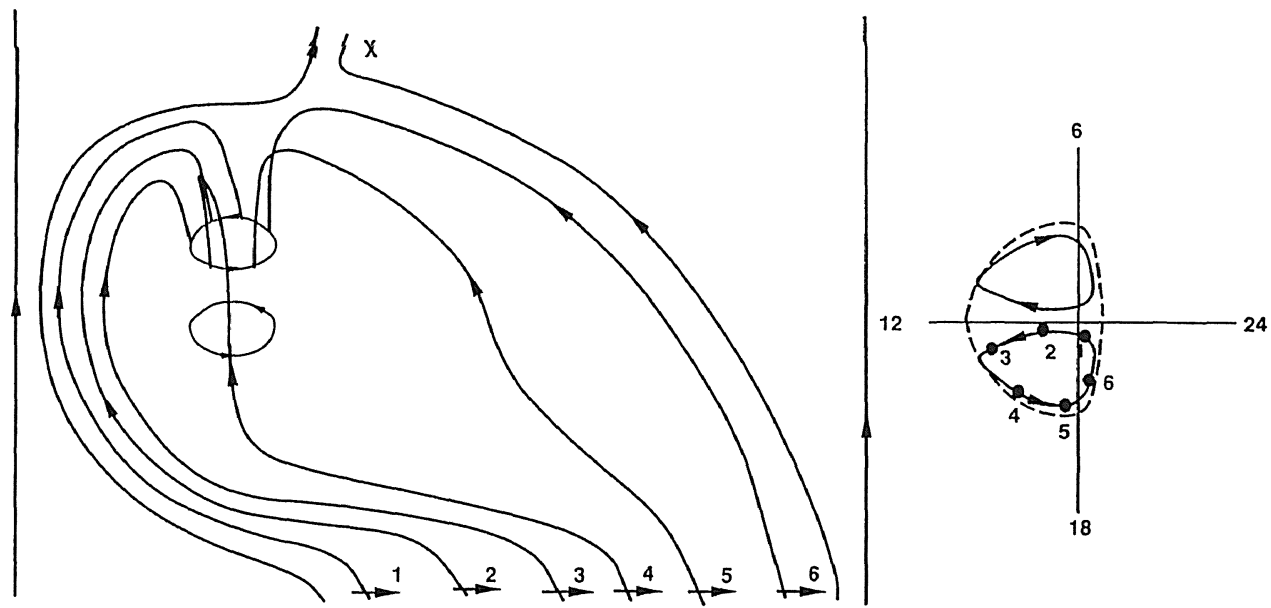

Fig. 3. Schematic representation of the magnetic field geometry and ionospheric flow resulting from connection between a northward IMF line and an already open field line that is part of the magnetotail. The field line labeled $\mathrm{X}$ must connect to a similarly distorted line draping the southern tail in order to avoid distortions in the IMF. 
laminar flow of magnetosheath plasma around the magnetopause produces a closed loop circulation in the ionosphere. A consequence of this flow and magnetic field geometry is that in the ionosphere, the plasma moves though a region (between points 6 and 1) where the magnetic flux tube geometry changes from one that crosses the equational plane in the tail to one that crosses the equatorial plane on the dayside. In general field lines may cross the equtorial plane on both sides of the magnetosphere, thus producing a two-cell convection pattern in the ionosphere whose asymmetric cell sizes are dependent on the ratio $B_{y} / B_{z}$ (LYONS, 1985). The distorted flux tube, labeled " $X$ " in Fig. 3, has a counterpart from similar electric and magnetic field configurations in the southern hemisphere. These distorted flux tubes must be removed by current flow in the solar wind so that no imprint of the magnetosphere is left in the frozen-in solar wind flow. For this configuratiion of the electric and magnetic fields no electric field is applied across the plasma sheet and the boundary enclosing the region of open field lines is an equipotential.

DUNGEY (1963) described a magnetic field geometry that produces an ionospheric convection pattern entirely on closed field lines for northward IMF. It is shown schematically in Fig. 4. As pointed out by RUSSELL (1972), the magnetic field geometry appears unlikely unless the IMF $y$-component is identically zero but it is included here for completeness. Again there are no merging or reconnection regions in this ionospheric flow pattern but it differs in a number of ways from that described previously as viscous interaction. In this picture, the magnetic field line geometry associated with the antisunward flowing plasma in the low latitude boundary layer is such that the antisunward flow near the nose of the magnetopause is associated with sunward flow at high latitudes. Antisunward flow further down the flanks of the low latitude boundary layer is associated with antisunward flow and perhaps sunward flow in the ionosphere, as is also the case of the previous described "viscous interaction" driver. For this configuration of electric and magnetic fields, the closed loop circulation in the ionosphere is associated with a continuous antisunward flow in the low latitude boundary layer. There is a region in the ionosphere (between points 6 and 1) across which the magnetic field geometry changes from being closed in the tail to being closed on the dayside.

If the convection pattern is not entirely on closed field lines, this implies that for northward IMF there exists an ionospheric signature of a merging region where the
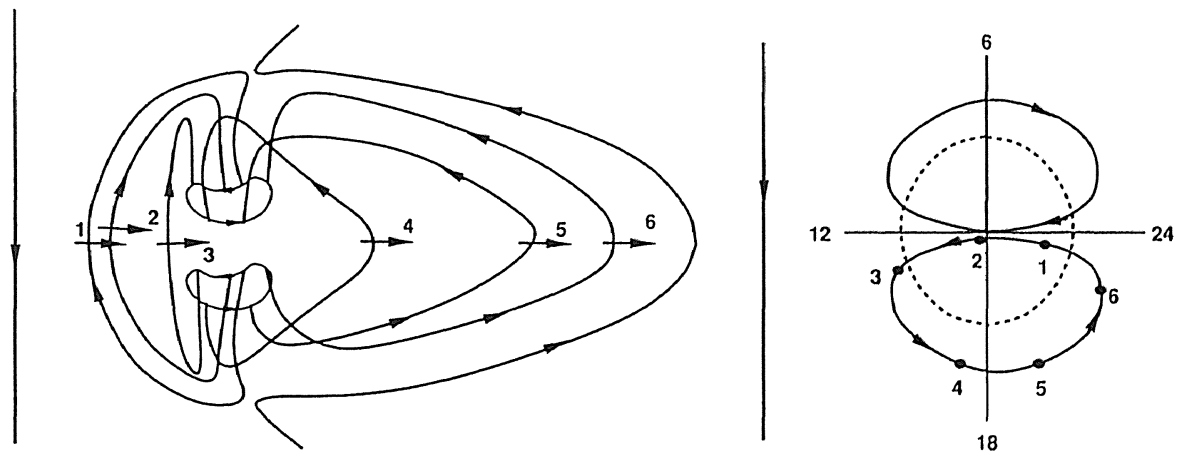

Fig. 4. Schematic representation of the magnetic field geometry and ionospheric flow resulting from simulataneous connection to a northward IMF in the northern and southern hemispheres. 
ionospheric plasma moves from closed to open field lines. The models of LYONS (1985) and CROOKER (1988) both contain such a region. The model of LYONS (1985) implies that the ionospheric signature of this region is located on the nightside of a region of sunward convection at high latitudes. CROOKER (1988) also shows that while the ionospheric projection of the merging region may have a more complex shape that the arc of a circle, it still resides on the nightside of the region of sunward convection. As noted previously, the location of a reconnection region in the ionosphere is not discussed by these models, but inspection of Figs. 3 and 4 show that it may be located anywhere on the closed loop trajectory and that its location will be define by the magnetic field geometry. For illustrative purposes we consider a case in which a reconnection region maps to a location in the antisunward flow region and where the convective flow on closed field lines contains both antisunward and sunward flow components. The ionospheric flow and magnetic field configuration is shown in Fig. 5 where the merging region is shown lightly shaded and the reconnection region is heavily shaded. The flow on open field lines is easily understood in terms of the electric field and magnetic field topology associated with the so called lobe cell in Fig. 3. Since this configuration applies equally to the southern hemisphere, we invoke a region on the flanks of the magnetosphere where the open field lines from the northern and southern hemispheres have the required geometry for reconnection. The remaining antisunward flow on closed field lines is associated with antisunward flow in the low-latitude boundary layer where the reconnection process itself allows the momentum of the magnetosheath plasma to be applied on the closed field lines as in Fig. 4. If this boundary layer flow continues antisunward in a tail configuration that narrows in the equatorial plane then this flow will map into sunward flow on closed field lines in the ionosphere as illustrated in Fig. 1. We note that this configuration of magnetic and electric fields produces a region of closed magnetic field lines surrounded on each side by open field lines from the nothern and southern polar regions. In this configuration, which is shown schematically in Fig. 6, the tail may be thought of as bifurcated. In Fig. 5 an open field line labeled 1*

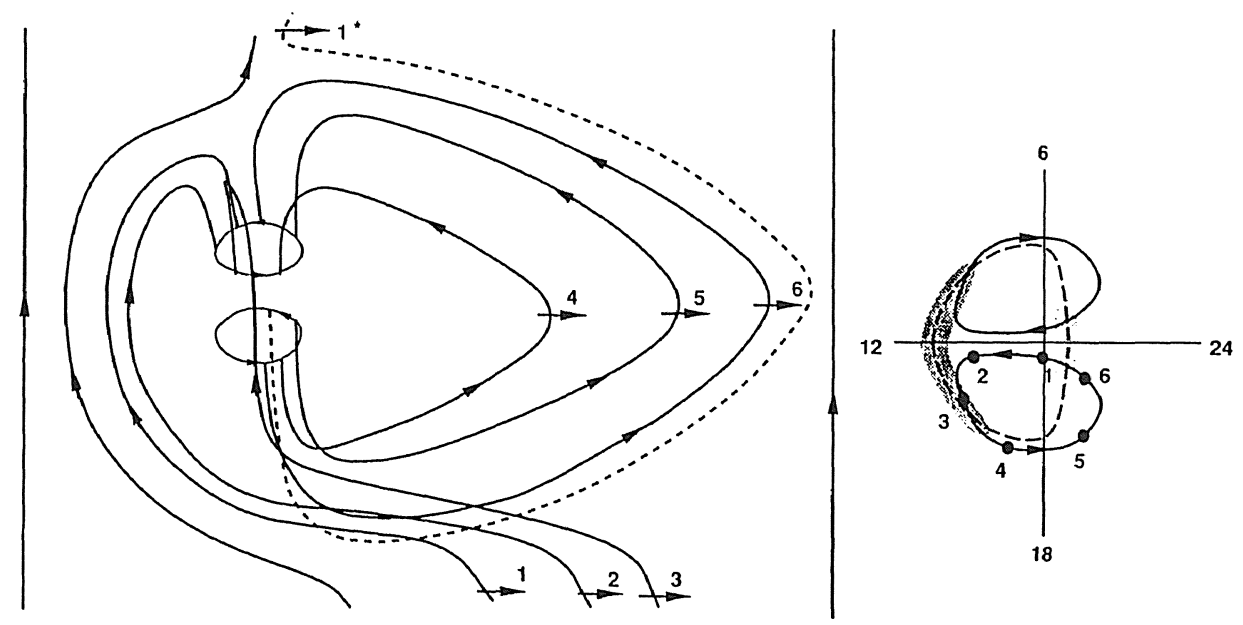

Fig. 5. Schematic representation of the magnetic field geometry and ionospheric flow resulting from connection of the northward IMF to closed field lines making up the magnetotail. 


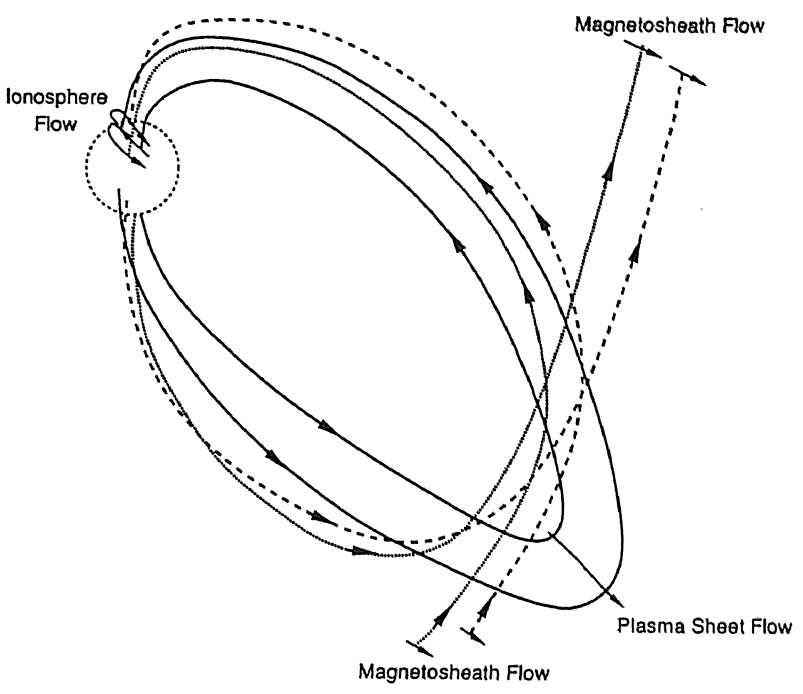

Fig. 6. Schematic illustration of the magnetic field configuration in a bifurcated tail. The open dotted field lines are draped around the observers side of the closed field lines while the open dashed field lines are draped behind the region of closed field lines. The small arrows show the direction of the convective flow in the magnetosphere and the ionosphere.

connecting to the southern high latitude region is shown to emphasize several points. If frozen-in flux is to be preserved then the magnetosheath flow associated with the flux tubes labeled 1 and $1^{*}$ must originate on the same IMF line and must eventually reside on the same IMF line in the tail. Since the open magnetic field lines containing 1 and $1^{*}$ convect around opposite sides of the magnetosphere, this requirement cannot be met if the reconnection region resides on the flanks of the magnetosphere as shown. Therefore, to ensure that beyond the immediate vicinity of the magnetosphere the frozen-in flux picture is preserved, we must allow plasma to move across open field lines while IMF flux tubes assemble their original order. Recall that appeal to a similar process was made to remove the distorted IMF line resulting from open flux recirculation in Fig. 3. This assumption, that regions exist where plasma can violate the frozen-in flux concepts across regions where the magnetic field topology does not change, is not in accordance with our original definitions of merging or reconnection and can only be removed by allowing the merging and reconnection regions to exist on field lines that exist in essentially the same location and have essentially the same stretched geometry in the tail. A field line stretching into the tail from this region may be either open or closed and plasma may move from open to closed field lines in the tail and from closed to open field lines in the high latitude cusp. A transition from one open field line to another actually occurs as a transition from open to closed to open field lines thus preserving our definition of merging and reconnection. The geomagnetic field lines that stretch into the tail produce an interesting non-conjugate effect in the expected particle emissions in the polar cap, as pointed out by LYONS (1985). Referring to Fig. 5, it can be seen that antisunward convection takes place on open magnetic flux tubes extending into the southern polar region that are continually reduced in volume. It is thus conceivable that particle precipitation will be seen at the feet of these flux tubes as the mirror points of the 
particles are continually lowered. Such precipitation features will appear in both hemisphere but will not be conjugate.

The convection patterns for northward IMF discussed here require that antisunward convection on closed or open magnetic field lines by observed in the ionosphere at the lowest latitudes directly influenced by the solar wind magnetosphere interaction. Such a requirement is not generally consistent with the observations illustrated during periods of northward IMF (BURKE et al., 1976; ZANETTI et al., 1985; HEELIS et al., 1986; HEPPNER and MAYNARD, 1987). This difficulty is removed in the models by an addition of two convection cells due to viscous interaction that appear on closed field lines at lower latitudes than the cells produced by direct connection with the IMF. This leads to a description of three or four convection cells with the lowest latitude flow having a sunward component. Such a description is certainly consistent with many of the observations. However, it neither addresses the distorted two-cell convection patterns, with which much of the data is also consistent (HEPPNER and MAYNARD, 1987), nor the evolution from two to three or four cell convection that takes place as the IMF changes from a southward to a northward orientation.

\section{Changes from Southward to Northward IMF}

The models of CROOKER $(1979,1988)$, LYONS (1985), and REIFF and BURCH (1986) all describe the evolution of an ionospheric convection pattern as the IMF changes from southward to northward. The models of CROOKER $(1979,1988)$ and LYONS $(1985)$ address only the convection driven by direct connection between the geomagnetic field and the IMF. In the model of LYONS (1985), the polar cap convection pattern changes from a configuration in which antisunward flow converges to a point on the nightside boundary to one in which sunward flow diverges from a point on the nightside boundary as the IMF changes from southward to northward. In the model of CROOKER (1988) a similar change in the orientation of the IMF produces a rotation of the merging region around the polar cap boundary from the dayside to the nightside. The sense of rotation is determined by the sign of the y-component of the IMF. The rotation of the convection pattern and the configuration implied for northward IMF is very similar to the insight gained from the field addition model of LYONS. The model convection pattern shows the ionospheric flow has an antisunward component at lowest latitudes. The observations, however, usualy show that the lowest latitude flows are sunward. The distorted two-cell patterns of HEPPNER and MAYNARD (1987) require that one cell intrude into and bifurcate the other. No such behavior is predicted by the two models discussed here. The model of REIFF and BURCH (1986) includes a contribution to the ionospheric convection pattern from viscous interaction. In their model the transition from southward to northward IMF may produce two cells circulating in opposite senses, or a lobe cell and a so-called reconnection cell circulating in the same sense. While the formation of the lobe and reconnection cell is well described, the transition to two lobe cells is less obvious.

A simple picture of the evolving convection pattern can be obtained by including the effects of both direct connection to the IMF and viscous interaction at all times (HEELIS and REIFF, 1991). We start by assuming that the magnetosheath plasma flow around the earths magnetosphere will always place a reconnection region in the magnetospheric tail for all orientations of the IMF. Thus, while the location of the convection reversal boundary may change, the ionospheric projection of the reconnection region will remain a long arc around its nightside. The motion of the ionospheric projection of the merging region may be taken 
from the recent work of CROOKER (1988) in which, as the IMF makes a transition from southward to northward, the merging region moves around the polar cap boundary. The sense of rotation of the merging region is determined by the sign of $B_{y}$. We will assume that, within the polar cap, any asymmetry in the geometry of the convection cells seen in the northern hemisphere is reversed in the southern hemisphere. Elsewhere, it is assumed that the closed geomagnetic field lines do not experience any severe distortion and thus ionospheric flows on closed field lines must have the same form in both hemispheres. The model of CROOKER (1988) requires that the merging region signature rotate in the opposite sense in the northern and southern hemispheres. This will produce considerable distortion in the closed field lines near the merging region that could be alleviated by requiring the merging region to expand or split and move dawnward and duskward as the MMF changes from sourthward to northward. With this hypothesis Fig. 7 shows the evolution of the northern hemisphere ionospheric convection pattern as the IMF changes from southward to northward with $B_{y}$ positive. A viscous interaction driver will produce a distortion of the convection cells produced by connection with the IMF. This model suggests that an expanded merging region would have enhanced flows across it near dawn and dusk when $B_{z} \approx 0$. Such regions of preferred flow across the boundary have been recently reported by MOSES et al. (1988). As the IMF becomes more northward we assume that open field lines emanating from the northern polar cap will traverse both flanks of the magnetosphere. At least two convection cells will then appear in the polar cap, as shown. Pursuing the split merging region hypothesis, the

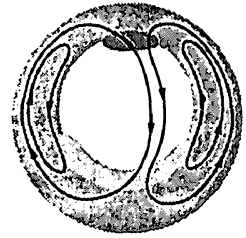

a

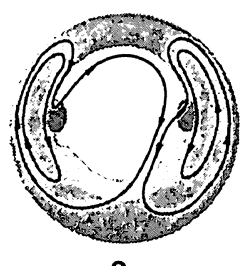

C

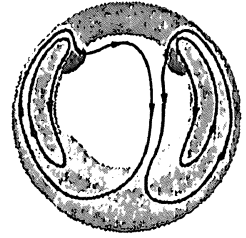

b

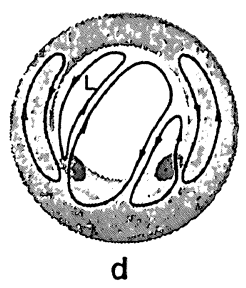

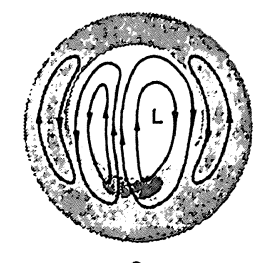

e

Fig. 7. Schematic representation of the evolution of ionospheric flow that results from two electric field sources when the IMF changes from southward to northward. 
formation of a small convection cell displaced to the dawnside of the nighttime polar cap may result from the magnetosheath flow associated with the rapidly shortened flux tubes that extend around the tail and into the southern hemisphere (see $1^{*}$ in Fig. 5). Without any bifurcation of the tail lobe, a large distorted cell similar in many respects to those illustrated by HEPPNER and MAYNARD (1987) is seen. When the merging and reconnection regions overlap lobe cell convection (labeled L) can occur.

\section{Conclusions}

We have described the configuration of plasma flow and magnetic field that can be used to interpret high latitude ionospheric convection measurements. It is emphasized that a simple examination of the data requires that the role of direct connection with the IMF and low-latitude boundary layer flows be considered as sources of the electric field. If both these drivers are considered then multiple cell and distorted two-cell convection patterns should be expected. Future challenges lie in reconciling observations with the orientation and evolution of the convection pattern that can be predicted from the conceptual models we have described.

This work is supported by NASA grants NAG 5-305 and NAG 5-306, and by Air Force Geophysics Laboratory contract F19628-90K-0001 to the University of Texas at Dallas.

\section{REFERENCE}

Burke, W. J., M. C. Kelley, R. C. Sagalyn, M. Smiddy, and S. T. Lai, Polar cap electric field structures with a northward interplanetary magnetic field, Geophys. Res. Lett., 6, 21, 1979.

Crooker, N. U., Dayside merging and cusp geometry, J. Geophys. Res., 84, 951, 1979.

CROOKER, N. U., Mapping the merging potential from the magnetopause to the ionosphere through the dayside cusp. J. Geophys. Res., 93, 7338-7344, 1988.

Dungey, J. W., The structure of the exosphere or adventures in velocity space, in Geophysics, The Earth's Environment, edited by C. DeWitt, J. Hiebolt, A. Lebeau, Gordon and Breach, New York, 1963.

Harel, M., R. A. Wolf, P. H. Reiff, R. W. Spiro, W. J. Burke, F. J. Rich, and M. Smiddy, Quantitative simulation of a magnetospheric substorm, I. Model logic and overview, J. Geophys. Res., 86, 2217, 1981.

HeElis, R. A., The effects of interplanetary magnetic field orientation on dayside high latitude convection, J. Geophys. Res., 89, 2873, 1984.

Heelis, R. A., P. H. Reiff, J. D. Winningham, and W. B. Hanson, Ionospheric convection signatures observed by DE-2 during northward interplanetary magnetic field, J. Geophys. Res., 91, 5817-5830, 1986.

Heelis, R. A. and P. H. ReIfF, The ionospheric convection pattern response to changes in IMF orientation, J. Geophys. Res., submitted, 1991.

Heikkila, W. J., Current sheet crossings in the distant magnetotail., Geophys. Res. Lett., 15, $299,1988$.

Heppner, J. P., Empirical models of high latitude electric fields, J. Geophys. Res., 82, 1115, 1977.

Heppner, J. P. and N. C. Maynard, Empirical high latitude electric field models, J. Geophys. Res., 92, 4467-4489, 1987.

Holt, J. M., R. H. WAND, J. V. Evans, and W. L. Oliver, Empirical models for the plasma convection at high latitudes from Millstone Hill observations, J. Geophys. Res., 92, 203-212, 1987.

Lockwood, M., S., W. H. Cowley, and M. P. FreEMAN, The excitation of plasma convection in the highlatitude ionosphere, J. Geophys. Res., 95, 7961-7972, 1990.

Lyons, L. R., A simple model for polar cap convection patterns and generation of $\theta$ auroras, J. Geophys. Res., 90, 1561, 1985.

Moses, J. J., G. L. Siscoe, R. A. Heelis, and J. D. Winningham. A model for multiple throat structures in the polar cap flow entry region, J. Geophys. Res., 93, 5741-5759, 1988. 
ReIfF, P. H. and L. J. BurCH, IMF $B_{y}$-Dependent plasma flow and Birkeland currents in the dayside magnetosphere, 2. A global model for northward and southward IMF, J. Geophys. Res., 90, 1595, 1985.

Russell, C. T., The configuration of the magnetosphere, in Critical Problems of Magnetospheric Physics, pp. 1-16, edited by E. R. Dryer, Jr., National Academy of Science, Washington, D.C., 1972.

Siscoe, G. L. and T. S. HuAng, Polar cap inflation and deflation, J. Geophys. Res., 90, 543-547, 1985.

ZanetTi, L. J., T. A. Potemra, T. IIJMA, W. BaumjohanN, and P. F. Bythrow, Ionospheric and Birkeland current distributions for northward interplanetary magnetic field, J. Geophys. Res., 89, 7458, 1984. 